\title{
VASCULAR FLORA OF OLD CEMETERIES TRANSFORMED INTO PARKS IN POZNAŃ
}

\author{
Aneta CZarna \\ A. Czarna, Department of Botany, Poznań University of Life Sciences, Wojska Polskiego 71 C, 60-625 \\ Poznań, Poland, e-mail: czarna@up.poznan.pl
}

(Received: November 23, 2017. Accepted: December 20, 2017)

\begin{abstract}
AвSTRACT. In 2011-2012, the vascular flora of all the eight park-like old cemeteries in Poznań was studied. They are former Protestant cemeteries, transformed into parks after the World War 2. Each of them was investigated in both spring and summer. In total, 334 species of vascular plants were found there, including 62 in the tree layer, 91 in the shrub layer, and 181 in the herb layer. Their vascular flora includes 173 taxa $(51.8 \%$ of the total) introduced into cultivation, most of them in the shrub layer (70 species, i.e. 20.9\%). Among plants with symbolic meanings, 52 species are in the tree and shrub layer, and 34 in the herb layer. Currently the symbolism of cemetery plants is of little significance, and they are planted mostly for ornament. Funeral plants in the investigated cemeteries are represented by 74 species. These include 15 species of geophytes, which are indicator plants (phytoindicators) of former cemeteries: Gagea arvensis, G. lutea, Galanthus nivalis, Hemerocallis fulva, Muscari botryoides, Narcissus poëticus, N. pseudonarcissus, Ornithogalum boucheanum, O. nutans, O. umbellatum, Puschkinia scilloides, Saponaria officinalis, Scilla sibirica, Tulipa gesneriana, and T. sylvestris.
\end{abstract}

Key words: Poznań, old cemeteries, vascular plants, funeral plants, ergasiophytes

\section{INTRODUCTION}

The aesthetic role of cemeteries increased in the $19^{\text {th }}$ century, when the concepts of cemetery parks, cemetery gardens or rural cemeteries were created. Plants started to play a major role in the cemetery space. Cemetery planning involved gardeners, landscape architects. Tall vegetation, shrubs, lawns, and flowers appeared in cemeteries (TANAś 2008). Because of the landscape cemetery concept, they are ideal for strolls and contemplation.

In Poland, old cemeteries can be transformed into parks 40 years after the last burial (NIEMIRSKI 1973). Thanks to the existence of parks at the sites of former cemeteries, they are cared for and maintained, and thus are easy to distinguish in the landscape. They are of both historical and environmental value.

The cemeteries where the proportion of tall vegetation is high are an important link in the system of urban green areas. Their extensive green spaces undoubtedly affect the microclimate of urban districts, and can also play an important visual role in urban landscape (NIEMIRSKI 1973). According to BorCZ (2002), cemeteries can be classified as urban green areas because they include groups of trees, alleys, vegetation planted at cemetery edges, solitary trees, and continuously intensively flowering plants.

SieWNiaK \& MitKowsKa (1998) report that irrespective of the epoch and geographic region, cemeteries fulfilling their major function (as burial sites) nearly always became also green areas. Their boundaries are well-defined, so they are like gardens of special type, always with emphasis on the atmosphere of silence, reflection, and meditation, usually with many sacral objects.

Municipal cemeteries, as wooded habitats, are classified also as urban green areas (SIEWNIAK \& MitKOWSKA 1998, DĄBSKI \& OlEś 2006). The oldest cemeteries are habitat islands in urban and suburban districts, often with old, valuable trees, a well-developed shrub layer, and interesting funeral plants in the herb layer. Before the World War 2, Hempelmann (1927) noted that individual lanes in cemeteries, to improve orientation, should be planted with contrasting tree species. Then the visitors would easily find their way even at large cemeteries.

Cemeteries are some of the most valuable forms of our cultural heritage. Now they also reflect the 
level of tolerance and sensitivity of the current generations in relation to other cultures or nationalities. This applies e.g. to abandoned German cemeteries (RYDZEWSKA et al. 2011).

In Poznan, the flora of several cemeteries has already been studied: historical cemeteries in Świerczewo (CZARna 2005) and the Citadel (CZARna 2016), and currently used ones: Górczyn Cemetery, Nowina Cemetery in Jeżyce, Corpus Christi Cemetery in Bluszczowa St., and John Vianney Parish Cemetery in Lutycka St. (CZARna et al. 2011).

Before the World War 2, cemeteries were used for burial but in the course of years they acquired the form of specific gardens. The plants were selected carefully, in a planned way, to create a complete composition. This is reflected in the current tree layer of vegetation in these cemeteries (transformed into parks), as the trees have reached maturity.

This study was aimed to analyse the taxonomic composition of vascular plants in park-like old cemeteries in Poznań and to verify the hypothesis that in park-like cemeteries the majority of funeral plants (cemetery phytoindicators) are geophytes.

\section{MATERIAL AND METHODS}

In 2011-2012, floristic investigations were conducted in eight park-like cemeteries. They are former Protestant cemeteries, transformed into parks after the World War 2. Each of them was investigated in both spring and summer (Table 1).

Names of spontaneously occurring species follow (Mirek et al. 2002), while names of cultivated species follow (GAWrYś 2008).

To determine the ground cover of species, a 7-degree scale of frequency was used: $\mathrm{R}=$ negligible (1-2 plants); $+=$ several plants; $1=$ very rare (covering $1-5 \%$ of cemetery area excluding graves); $2=$ rare $(5-25 \%) ; 3=$ moderately frequent $(25-50 \%)$; $4=$ frequent $(50-75 \%) ; 5=$ common $(75-100 \%)$.

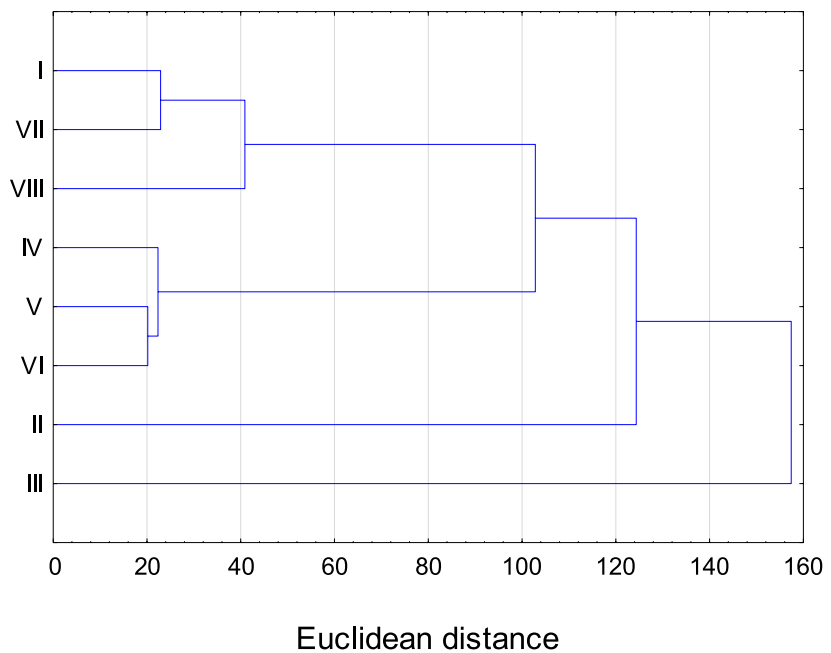

Fig. 1. General floristic similarity of the studied cemeteries visualized using cluster analysis Explanations - see Table 2.

Abbreviation "cul" indicates that the species was planted (long ago or recently).

Geographical-historical classification follow those proposed by THellung (1915) with some modifications. Ergasiophytes were defined as alien species of cultivated plants introduced intentionally by humans and later neglected, which are permanently naturalised generatively or vegetatively in parks, gardens or cemeteries, but do not invade neighbouring habitats.

While Raunkiaer's plant life-forms are classified mostly according to RUTKOWSKI (1998), ZARZYCKI et al. (2002). Species new to Poland were determined in comparison to MireK et al. (2002), species new to Wielkopolska were distinguished in comparison to SzULCZEWSKI (1951), and species new to Poznań were identified in reference to JACKOWIAK (1990). Plants with symbolic meanings were determined on the basis of KopalińsKi (1985), ZıóŁKOWSKa (1988), Kobielus (2006), Kossak (2017).

Table 1. List of the investigated park-like old cemeteries in Poznań

\begin{tabular}{|c|c|c|c|c|c|c|}
\hline No. & Polish name & Street & Denomination & Graves & Use & Date of research \\
\hline I & Malta & Świętojańska & Catholic & none & mown & $\begin{array}{l}24.04 .2012 \\
24.07 .2012\end{array}$ \\
\hline II & Park Górczyński & Ostrobramska & Protestant & none & mown & $\begin{array}{l}20.04 .2012 \\
26.07 .2012\end{array}$ \\
\hline III & Park K. Marcinkowskiego & Towarowa & Protestant & none & mown & $\begin{array}{l}04.04 .2011 \\
23.07 .2012\end{array}$ \\
\hline IV & Park Lubuski & Królowej Jadwigi & Protestant & none & mown & $\begin{array}{l}22.04 .2012 \\
23.07 .2012\end{array}$ \\
\hline $\mathrm{V}$ & Park G. Manitiusa & Grunwaldzka & Protestant & none & mown & $\begin{array}{l}02.04 .2011 \\
30.08 .2011\end{array}$ \\
\hline VI & Park. im. Gen. J. H. Dąbrowskiego & Ogrodowa & Protestant & none & mown & $\begin{array}{l}22.04 .2012 \\
23.07 .2012\end{array}$ \\
\hline VII & Plac Kosynierów & Słowiańska & Protestant & 1 grave & mown & $\begin{array}{l}22.04 .2012 \\
30.07 .2012\end{array}$ \\
\hline VIII & Park near Hotel Vivaldi & Winogrady & Protestant & none & no management & $\begin{array}{l}22.04 .2012 \\
24.07 .2012\end{array}$ \\
\hline
\end{tabular}


The species that occurred in 2-3 layers of vegetation in the studied cemeteries, were counted twice or thrice in the classification of life-forms and geographical-historical groups, i.e. as they occurred.

General floristic similarity of the studied cemeteries was visualized using cluster analysis. The dendrogram was generated by Statistica software on the basis of Euclidean distances and the unweighted pair-group average. The dendrogram (Fig. 1) was generated on the basis of species occurrence assessed using the Braun-Blanquet scale. The scale was transformed into a numerical scale as follows: $r$ into $1,+$ into 2,1 into 3,2 into 4, 3 into 5, 4 into 6 , and 5 into 7. Each species was used in the analysis only once, even if it occurred in many layers.

\section{RESULTS}

In all the eight park-like old cemeteries in Poznań, 334 species of vascular plants were found. The tree layer consisted of 62 species, the shrub layer of 91, and the herb layer of 181 (Table 2). Numbers of recorded species were the highest in cemeteries III (155 species), I (119), and VIII (108), whereas the lowest in cemeteries VI (58) and V (66) (Table 3).

In general, the highest number of species represented frequency class "+" (341 records), followed by classes "1" (226) and "R" (121) (Table 3). Only one species (Aesculus hippocastanum) in one cemetery (VI) was included in the highest frequency class " 5 ". Also frequency class "4" was represented by only one species (Lolium multiflorum), in the same cemetery (VI). Plants of frequency class " 3 " were found in cemeteries III (1 species), IV (5), V (6), VII (1), and VIII (3). The lowest frequency class, "R", was represented by the largest numbers of species in cemeteries I, III, IV, and VI. Frequency classes "2" and "3" included the largest numbers of species in cemetery III.

Raunkiaer's life-forms are adaptations to survival in unfavourable periods, such as winter and droughts. Vascular plants of park-like cemeteries in Poznań represent six life-forms: megaphanerophytes, nanophanerophytes, chamaephytes, geophytes, therophytes, and hemicryptophytes. Among

Table 2. Occurrence of vascular plants in park-like cemeteries in Poznań and their classification into life-forms (LF), geographical-historical groups (GH), symbolic plants (S), and funeral plants (F)

\begin{tabular}{|c|c|c|c|c|c|c|c|c|c|c|c|}
\hline \multirow[b]{2}{*}{ Species } & \multicolumn{8}{|c|}{ Cemetery } & \multirow[b]{2}{*}{ LF } & \multirow[b]{2}{*}{$\mathrm{GH}$} & \multirow{2}{*}{$\begin{array}{c}\text { Symbolic } \\
(\mathrm{S}) \text { and } \\
\text { funeral }(\mathrm{F}) \\
\text { plants }\end{array}$} \\
\hline & I & II & III & IV & $\mathrm{V}$ & VI & VII & VIII & & & \\
\hline \multicolumn{12}{|c|}{ Tree layer } \\
\hline Abies concolor (Gordon \& Glend.) Lindl. ex Hildebr. & - & - & 2 cul & $1 \mathrm{cul}$ & $1 \mathrm{cul}$ & - & - & - & F1 & ErW & $\mathrm{S}$ \\
\hline Acer campestre L. & - & $1 \mathrm{cul}$ & $1 \mathrm{cul}$ & - & - & - & - & - & $\mathrm{F} 1$ & Ap2/Sp2 & $S$ \\
\hline Acer negundo L. & - & - & $1 \mathrm{cul}$ & - & - & - & $1 \mathrm{cul}$ & - & $\mathrm{F} 1$ & $\mathrm{Kn}$ & $S$ \\
\hline Acer platanoides L. & 2 cul & 2 cul & 2 cul & 3 cul & $2 \mathrm{cul}$ & $1 \mathrm{cul}$ & $3 \mathrm{cul}$ & 3 cul & $\mathrm{F} 1$ & Ap2 & $S, F$ \\
\hline Acer pseudoplatanus L. & $1 \mathrm{cul}$ & $1 \mathrm{cul}$ & $1 \mathrm{cul}$ & - & $1 \mathrm{cul}$ & $1 \mathrm{cul}$ & - & $1 \mathrm{cul}$ & $\mathrm{F} 1$ & Ap2 & $\mathrm{S}, \mathrm{F}$ \\
\hline Acer saccharinum L. & - & - & - & $1 \mathrm{cul}$ & - & - & - & $1 \mathrm{cul}$ & $\mathrm{F} 1$ & ErW & $S$ \\
\hline Acer tataricum L. & - & - & $1 \mathrm{cul}$ & $1 \mathrm{cul}$ & - & - & - & - & $\mathrm{F} 1$ & ErW & S \\
\hline Aesculus carnea Hayne & - & - & $1 \mathrm{cul}$ & - & - & - & - & - & $\mathrm{F} 1$ & ErW & $S$ \\
\hline Aesculus hippocastanum L. & $1 \mathrm{cul}$ & - & $1 \mathrm{cul}$ & 3 cul & $1 \mathrm{cul}$ & $4 \mathrm{cul}$ & - & $2 \mathrm{cul}$ & $\mathrm{F} 1$ & $\mathrm{Kn}$ & $S, F$ \\
\hline Ailanthus altissima Swingle & $1 \mathrm{cul}$ & - & $1 \mathrm{cul}$ & - & - & - & - & - & $\mathrm{F} 1$ & $\mathrm{Kn}$ & \\
\hline Betula pendula Roth & - & - & $1 \mathrm{cul}$ & - & - & - & - & - & $\mathrm{F} 1$ & Ap2 & S \\
\hline Carpinus betulus L. & $1 \mathrm{cul}$ & $1 \mathrm{cul}$ & $1 \mathrm{cul}$ & $1 \mathrm{cul}$ & - & - & - & - & $\mathrm{F} 1$ & Sp2 & \\
\hline Catalpa bignonioides Walter & - & - & $1 \mathrm{cul}$ & - & - & - & - & - & $\mathrm{F} 1$ & ErW & \\
\hline Cerasus mahaleb (L.) Mill. & - & - & $1 \mathrm{cul}$ & - & - & - & - & - & $\mathrm{F} 1$ & $\mathrm{Kn}$ & \\
\hline Crataegus $\times$ media Bechst. & - & - & - & - & - & $1 \mathrm{cul}$ & - & - & $\mathrm{F} 2$ & ErW & S \\
\hline Crataegus monogyna Jacq. & - & - & $1 \mathrm{cul}$ & - & - & - & - & - & $\mathrm{F} 2$ & Ap2/Sp2 & S \\
\hline Fagus sylvatica L. & $1 \mathrm{cul}$ & $1 \mathrm{cul}$ & $1 \mathrm{cul}$ & - & - & - & - & - & $\mathrm{F} 1$ & Ap2/Sp2 & S \\
\hline Fagus sylvatica L. 'Pendula' & - & - & - & - & $1 \mathrm{cul}$ & - & - & - & $\mathrm{F} 1$ & ErW & $S, F$ \\
\hline Fraxinus excelsior L. & 2 cul & $2 \mathrm{cul}$ & $1 \mathrm{cul}$ & $1 \mathrm{cul}$ & $2 \mathrm{cul}$ & $1 \mathrm{cul}$ & $1 \mathrm{cul}$ & $1 \mathrm{cul}$ & $\mathrm{F} 1$ & Ap2 & $\mathrm{S}, \mathrm{F}$ \\
\hline Fraxinus excelsior L. 'Diversifolia' & - & - & - & - & - & $1 \mathrm{cul}$ & - & - & $\mathrm{F} 1$ & ErW & $\mathrm{S}$, \\
\hline Fraxinus excelsior L. 'Pendula' & - & $1 \mathrm{cul}$ & - & - & $1 \mathrm{cul}$ & - & - & - & $\mathrm{F} 1$ & ErW & $\mathrm{S}, \mathrm{F}$ \\
\hline Fraxinus pennsylvanica Marshall & - & - & $1 \mathrm{cul}$ & - & - & - & - & - & $\mathrm{F} 1$ & $\mathrm{Kn}$ & $\mathrm{S}$ \\
\hline Gleditsia triacanthos $\mathrm{L}$. & - & - & $1 \mathrm{cul}$ & - & - & - & - & - & $\mathrm{F} 1$ & ErW & \\
\hline Hedera helix L. & - & - & - & - & - & - & - & $1 \mathrm{cul}$ & $\mathrm{C}$ & $\mathrm{Kn}$ & $\mathrm{S}, \mathrm{F}$ \\
\hline Juglans regia $\mathrm{L}$. & $1 \mathrm{cul}$ & - & - & - & - & - & - & - & $\mathrm{F} 1$ & $\mathrm{Kn}$ & $\mathrm{S}$ \\
\hline Malus domestica Borkh. & - & - & $1 \mathrm{cul}$ & $1 \mathrm{cul}$ & - & - & - & - & $\mathrm{F} 1$ & $\mathrm{Kn}$ & $\mathrm{S}$ \\
\hline Malus $\times$ oxysepala Czarna \& Nowińska & - & - & 1 & - & - & - & - & - & F1 & Ap $1 \times$ ErG & $\mathrm{S}$ \\
\hline Malus $\times$ purpurea Rehder & $1 \mathrm{cul}$ & - & $1 \mathrm{cul}$ & $1 \mathrm{cul}$ & - & - & - & - & $\mathrm{F} 1$ & ErW & S \\
\hline Morus alba L. & - & - & - & $1 \mathrm{cul}$ & - & - & - & - & $\mathrm{F} 1$ & ErW & S \\
\hline Morus nigra L. & $1 \mathrm{cul}$ & - & - & - & - & - & - & - & $\mathrm{F} 1$ & ErW & $\mathrm{S}$ \\
\hline Padus serotina (Ehrh.) Borkh. & - & $1 \mathrm{cul}$ & - & - & - & - & - & - & $\mathrm{F} 1$ & $\mathrm{Kn}$ & $S$ \\
\hline Picea abies (L.) H. Karst. & - & - & - & $1 \mathrm{cul}$ & - & - & - & - & F1 & $\mathrm{Kn}$ & $\mathrm{S}$ \\
\hline Picea pungens Engelm. & $1 \mathrm{cul}$ & - & $1 \mathrm{cul}$ & - & $1 \mathrm{cul}$ & - & $1 \mathrm{cul}$ & - & $\mathrm{F} 1$ & ErG & $S$ \\
\hline
\end{tabular}




\begin{tabular}{|c|c|c|c|c|c|c|c|c|c|c|c|}
\hline \multirow[b]{2}{*}{ Species } & \multicolumn{8}{|c|}{ Cemetery } & \multirow[b]{2}{*}{ LF } & \multirow[b]{2}{*}{$\mathrm{GH}$} & \multirow{2}{*}{$\begin{array}{c}\text { Symbolic } \\
(\mathrm{S}) \text { and } \\
\text { funeral }(\mathrm{F}) \\
\text { plants }\end{array}$} \\
\hline & I & II & III & IV & $\mathrm{V}$ & VI & VII & VIII & & & \\
\hline Pinus strobus L. & - & $1 \mathrm{cul}$ & - & - & - & - & - & - & F1 & $\mathrm{Kn}$ & S \\
\hline Platanus $\times$ hispanica Mill. ex Műnchh. & - & $3 \mathrm{cul}$ & $1 \mathrm{cul}$ & $1 \mathrm{cul}$ & $1 \mathrm{cul}$ & - & - & - & $\mathrm{F} 1$ & ErW & \\
\hline Populus alba L. & $1 \mathrm{cul}$ & $1 \mathrm{cul}$ & $1 \mathrm{cul}$ & - & - & - & $1 \mathrm{cul}$ & $1 \mathrm{cul}$ & $\mathrm{F} 1$ & Ap2 & S \\
\hline Populus $\times$ canadensis Moench & - & - & $1 \mathrm{cul}$ & - & - & - & - & $1 \mathrm{cul}$ & $\mathrm{F} 1$ & ErW & $S$ \\
\hline Populus nigra L. & - & - & $1 \mathrm{cul}$ & $1 \mathrm{cul}$ & - & - & - & - & F1 & Ap2/Sp2 & S \\
\hline Populus tremula L. & - & - & $1 \mathrm{cul}$ & $1 \mathrm{cul}$ & - & - & $1 \mathrm{cul}$ & $1 \mathrm{cul}$ & $\mathrm{F} 1$ & Ap2 & S \\
\hline Prunus cerasifera Ehrh. & $1 \mathrm{cul}$ & - & $1 \mathrm{cul}$ & $2 \mathrm{cul}$ & - & - & - & - & F1 & $\mathrm{Kn}$ & S \\
\hline Prunus cerasifera Ehrh. 'Atropurpurea' & - & - & $1 \mathrm{cul}$ & $1 \mathrm{cul}$ & - & - & - & - & $\mathrm{F} 1$ & $\mathrm{Kn}$ & $S$ \\
\hline Prunus domestica L. & - & 1 & - & - & - & - & - & - & $\mathrm{F} 1$ & ErW & $\mathrm{S}$ \\
\hline Pseudotsuga menziesii (Mirb.) Franco & $1 \mathrm{cul}$ & 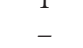 & $1 \mathrm{cul}$ & $1 \mathrm{cul}$ & - & - & - & - & $\mathrm{F} 1$ & $\mathrm{Kn}$ & \\
\hline Pyrus pyraster (L.) Burgsd. & - & - & $1 \mathrm{cul}$ & $1 \mathrm{cul}$ & - & - & - & $1 \mathrm{cul}$ & $\mathrm{F} 1$ & $\mathrm{Kn}$ & $S$ \\
\hline Quercus robur $\mathrm{L}$. & - & $2 \mathrm{cul}$ & $1 \mathrm{cul}$ & $2 \mathrm{cul}$ & $2 \mathrm{cul}$ & $2 \mathrm{cul}$ & - & - & $\mathrm{F} 1$ & Ap2/Sp2 & $\mathrm{S}, \mathrm{F}$ \\
\hline Quercus rubra L. & - & - & - & - & $1 \mathrm{cul}$ & - & - & - & $\mathrm{F} 1$ & $\mathrm{Kn}$ & $S, F$ \\
\hline Quercus robur $\times$ Q. petraea & - & - & - & - & $1 \mathrm{cul}$ & - & - & - & F1 & Ap2/Sp2 & S \\
\hline Rhamnus cathartica L. & - & - & 1 & 1 & - & - & - & - & F1 & $\mathrm{Ap} 2 / \mathrm{Sp} 2$ & \\
\hline Robinia pseudoacacia L. & $1 \mathrm{cul}$ & $1 \mathrm{cul}$ & $2 \mathrm{cul}$ & $1 \mathrm{cul}$ & - & $1 \mathrm{cul}$ & $1 \mathrm{cul}$ & $1 \mathrm{cul}$ & $\mathrm{F} 1$ & $\mathrm{Kn}$ & $S, F$ \\
\hline Salix alba L. & - & - & $1 \mathrm{cul}$ & - & - & - & - & - & $\mathrm{F} 1$ & Ap2 & $S$ \\
\hline Salix alba L. 'Tristis' & - & - & $1 \mathrm{cul}$ & - & - & - & - & - & $\mathrm{F} 1$ & ErW & $S, F$ \\
\hline Sophora japonica L. & - & - & $1 \mathrm{cul}$ & - & - & - & - & - & F1 & ErW & $\mathrm{F}$ \\
\hline Sorbus aucuparia L. emend. Hedl. & - & - & $1 \mathrm{cul}$ & - & - & - & - & - & F1 & Ap2/Sp2 & S \\
\hline Sorbus intermedia (Ehrh.) Pers. & - & - & $1 \mathrm{cul}$ & - & - & - & - & - & $\mathrm{F} 1$ & ErG & $S, F$ \\
\hline Thuja plicata Donn ex D. Don & - & $1 \mathrm{cul}$ & - & - & $1 \mathrm{cul}$ & - & - & - & $\mathrm{F} 1$ & ErW & S, F \\
\hline Tilia americana & - & - & $1 \mathrm{cul}$ & - & - & - & - & - & $\mathrm{F} 1$ & ErW & $S$ \\
\hline Tilia cordata Mill. & - & $2 \mathrm{cul}$ & - & $1 \mathrm{cul}$ & $3 \mathrm{cul}$ & $1 \mathrm{cul}$ & $1 \mathrm{cul}$ & $1 \mathrm{cul}$ & $\mathrm{F} 1$ & Ap2 & $S, F$ \\
\hline Tilia 'Euchlora' & $2 \mathrm{cul}$ & - & $1 \mathrm{cul}$ & $1 \mathrm{cul}$ & $3 \mathrm{cul}$ & - & - & - & $\mathrm{F} 1$ & ErW & $\mathrm{S}, \mathrm{F}$ \\
\hline Tilia platyphyllos Scop. & - & $3 \mathrm{cul}$ & $1 \mathrm{cul}$ & $1 \mathrm{cul}$ & $1 \mathrm{cul}$ & $1 \mathrm{cul}$ & - & $2 \mathrm{cul}$ & $\mathrm{F} 1$ & ErG & S, F \\
\hline Ulmus glabra Huds. & - & - & - & - & $1 \mathrm{cul}$ & - & - & - & $\mathrm{F} 1$ & Ap2 & S \\
\hline Ulmus laevis Pall. & $1 \mathrm{cul}$ & $1 \mathrm{cul}$ & $1 \mathrm{cul}$ & $2 \mathrm{cul}$ & $1 \mathrm{cul}$ & $1 \mathrm{cul}$ & $1 \mathrm{cul}$ & - & F1 & Ap2/Sp2 & $S$ \\
\hline Viscum album $\mathrm{L}$. & + & + & 1 & 1 & + & + & 1 & + & $\mathrm{C}$ & Ap1 & S \\
\hline \multicolumn{12}{|c|}{ Shrub layer } \\
\hline Abies concolor (Gordon \& Glend.) Lindl. ex Hildebr. & - & - & + cul & - & - & - & - & - & F1 & ErW & S \\
\hline Acer campestre L. & - & 1 & - & - & - & - & - & - & F1 & Ap2/Sp2 & S \\
\hline Acer platanoides L. & + & - & + & + & - & - & + & 1 & F1 & Apl & $S$ \\
\hline Acer pseudoplatanus $\mathrm{L}$. & - & + & - & - & - & - & - & + & $\mathrm{F} 1$ & Ap1 & S \\
\hline Aesculus hippocastanum L. & - & - & - & - & - & - & - & 2 & F1 & $\mathrm{Kn}$ & S \\
\hline Amelanchier spicata Lam. & - & $+\mathrm{cul}$ & - & - & - & - & - & $1 \mathrm{cul}$ & $\mathrm{F} 2$ & $\mathrm{Kn}$ & \\
\hline Berberis thunbergii DC. & - & - & $+\mathrm{cul}$ & - & - & - & $+\mathrm{cul}$ & - & $\mathrm{F} 2$ & ErW & S \\
\hline Berberis vulgaris L. & $+\mathrm{cul}$ & - & + cul & $1 \mathrm{cul}$ & - & - & - & - & $\mathrm{F} 2$ & Ap2/Sp2 & $S$ \\
\hline Betula pendula Roth & $+\mathrm{cul}$ & - & - & - & - & - & - & - & $\mathrm{F} 1$ & Ap2 & $S$ \\
\hline Caragana arborescens Lam. & - & - & + cul & - & - & - & - & - & $\mathrm{F} 2$ & ErG & F \\
\hline Carpinus betulus L. & - & $+\mathrm{cul}$ & - & - & - & - & - & - & F1 & Sp2 & \\
\hline Catalpa bignonioides Walter & - & - & - & + cul & - & - & - & - & $\mathrm{F} 1$ & ErW & \\
\hline Castanea sativa Mill. & + cul & - & - & - & - & - & - & - & $\mathrm{F} 1$ & ErW & S \\
\hline Cerasus avium (L.) Moench & - & + & - & - & - & - & - & - & $\mathrm{F} 1$ & $\mathrm{Kn}$ & $S, F$ \\
\hline Cerasus mahaleb (L.) Mill. & - & - & - & - & - & - & - & $+\mathrm{cul}$ & F1 & $\mathrm{Kn}$ & $\mathrm{F}$ \\
\hline Clematis vitalba $\mathrm{L}$. & - & - & - & - & $+\mathrm{cul}$ & - & - & $+\mathrm{cul}$ & $\mathrm{C}$ & $\mathrm{Kn}$ & $\mathrm{F}$ \\
\hline Cornus alba L. & - & - & - & - & - & $+\mathrm{cul}$ & - & - & $\mathrm{F} 2$ & ErW & \\
\hline Cornus mas L. & - & - & + cul & + cul & - & - & - & - & $\mathrm{F} 1$ & ErW & \\
\hline Corylus avellana L. & $1 \mathrm{cul}$ & - & - & - & - & - & - & - & $\mathrm{F} 2$ & $\mathrm{Sp} 2$ & $S$ \\
\hline Cotinus coggygria Scop. & - & - & - & - & - & $+\mathrm{cul}$ & - & - & $\mathrm{F} 2$ & ErW & \\
\hline Crataegus monogyna Jacq. & - & - & $+\mathrm{cul}$ & - & - & - & - & - & $\mathrm{F} 1$ & Ap2/Sp2 & S \\
\hline Euonymus europaea L. & - & - & + & + & - & - & - & + & $\mathrm{F} 2$ & Apl/Sp1 & \\
\hline Euonymus fortunei (Turcz.) Hand.-Mazz. & - & - & + cul & - & - & - & - & - & $\mathrm{F} 2$ & ErW & \\
\hline Fagus sylvatica $\mathrm{L}$. & - & $+\mathrm{cul}$ & - & + cul & - & - & - & - & F1 & Ap1/Sp1 & S \\
\hline Fagus sylvatica L. 'Atropurpurea' & - & - & - & + cul & - & + cul & - & - & F1 & ErW & S \\
\hline Forsythia $\times$ intermedia Zabel & $+\mathrm{cul}$ & $+\mathrm{cul}$ & + cul & $1 \mathrm{cul}$ & $+\mathrm{cul}$ & - & $+\mathrm{cul}$ & - & $\mathrm{F} 2$ & ErW & \\
\hline Fraxinus excelsior $\mathrm{L}$. & - & + & 1 & + & - & - & - & + & $\mathrm{F} 1$ & Ap1 & $S$ \\
\hline Ginkgo biloba L. & - & - & + cul & - & - & - & - & - & $\mathrm{F} 1$ & ErW & \\
\hline Hedera helix L. & - & - & - & - & - & - & - & $1 \mathrm{cul}$ & $\mathrm{C}$ & $\mathrm{Kn}$ & $S, F$ \\
\hline Juglans regia $\mathrm{L}$. & - & - & - & - & - & - & + & + & F1 & $\mathrm{Kn}$ & $\mathrm{S}$ \\
\hline Juniperus horizontalis Moench & - & - & - & $1 \mathrm{cul}$ & - & - & - & - & $\mathrm{F} 2$ & ErW & S, F \\
\hline Larix polonica Racib. & $+\mathrm{cul}$ & - & - & - & - & - & - & - & F1 & Sp2 & $S$ \\
\hline Laurocerasus officinalis Roem. & - & - & + cul & - & - & - & - & - & F2 & ErW & \\
\hline
\end{tabular}




\begin{tabular}{|c|c|c|c|c|c|c|c|c|c|c|c|}
\hline \multirow[b]{2}{*}{ Species } & \multicolumn{8}{|c|}{ Cemetery } & \multirow[b]{2}{*}{ LF } & \multirow[b]{2}{*}{$\mathrm{GH}$} & \multirow{2}{*}{$\begin{array}{c}\text { Symbolic } \\
(\mathrm{S}) \text { and } \\
\text { funeral }(\mathrm{F}) \\
\text { plants }\end{array}$} \\
\hline & I & II & III & IV & $\mathrm{V}$ & VI & VII & VIII & & & \\
\hline Ligustrum vulgare L. & + cul & $+\mathrm{cul}$ & + cul & + cul & - & - & - & + cul & $\mathrm{F} 2$ & ErW & F \\
\hline Lonicera $\times$ bella Zabel & - & + cul & + cul & - & - & - & - & - & $\mathrm{F} 2$ & ErW & \\
\hline Lonicera maackii (Rupr.) Herder & - & - & + cul & - & - & - & - & - & $\mathrm{F} 2$ & ErW & \\
\hline Lonicera tatarica $\mathrm{L}$. & - & + cul & - & + cul & + cul & - & - & - & $\mathrm{F} 2$ & ErW & \\
\hline Lonicera xylosteum $\mathrm{L}$. & - & - & + cul & - & + cul & - & - & - & $\mathrm{F} 2$ & Ap2/Sp2 & \\
\hline Lycium barbatum L. & - & - & + cul & $1 \mathrm{cul}$ & - & - & - & - & $\mathrm{F} 2$ & $\mathrm{Kn}$ & \\
\hline Mahonia aquifolium (Pursh) Nutt. & - & - & - & + cul & + cul & - & - & - & $\mathrm{F} 2$ & ErW & $\mathrm{S}, \mathrm{F}$ \\
\hline Malus baccata (L.) Borkh. & - & - & $1 \mathrm{cul}$ & - & - & - & - & - & $\mathrm{F} 1$ & ErW & $S$ \\
\hline Malus $\times$ purpurea Rehder & - & - & $1 \mathrm{cul}$ & - & - & - & - & - & $\mathrm{F} 1$ & ErW & $S$ \\
\hline Morus alba L. & - & - & - & - & - & - & - & + cul & $\mathrm{F} 1$ & ErW & $S$ \\
\hline Padus avium Mill. & - & - & - & + cul & - & - & - & - & $\mathrm{F} 2$ & Sp1 & $\mathrm{S}, \mathrm{F}$ \\
\hline Padus serotina (Ehrh.) Borkh. & - & - & - & - & - & - & - & + & $\mathrm{F} 2$ & Kn & $\mathrm{S}, \mathrm{F}$ \\
\hline $\begin{array}{l}\text { Parthenocissus quinquefolia (L.) Planch. in A. \& } \\
\text { C.DC. }\end{array}$ & + cul & - & - & - & - & - & - & - & $\mathrm{C}$ & ErW & $\mathrm{F}$ \\
\hline Persica vulgaris Mill. & + & - & - & - & - & - & - & - & $\mathrm{F} 2$ & ErG & \\
\hline Philadelphus coronarius L. & - & $1 \mathrm{cul}$ & $1 \mathrm{cul}$ & - & + cul & - & - & - & $\mathrm{F} 2$ & ErW & $\mathrm{F}$ \\
\hline Physocarpus opulifolius (L.) Maxim. & - & - & - & - & + cul & + cul & - & - & $\mathrm{F} 2$ & ErW & \\
\hline Picea abies (L.) H. Karst. & + cul & - & - & - & - & - & - & - & $\mathrm{F} 1$ & $\mathrm{Kn}$ & S \\
\hline Picea pungens Engelm. & + cul & - & + cul & - & - & - & - & - & F1 & ErW & S \\
\hline Pinus sylvestris L. & + cul & - & - & - & - & - & - & - & $\mathrm{F} 1$ & Ap2 & S \\
\hline Platanus $\times$ hispanica Münhchh & - & - & $1 \mathrm{cul}$ & + cul & + cul & - & - & - & $\mathrm{F} 1$ & ErW & \\
\hline Populus alba L. & - & - & - & - & - & - & - & + & F1 & Ap1 & $S$ \\
\hline Potentilla fruticosa $\mathrm{L}$. & - & - & $1 \mathrm{cul}$ & - & - & - & - & - & $\mathrm{F} 2$ & ErW & \\
\hline Prunus cerasifera Ehrh. & + & - & + & - & - & - & - & - & $\mathrm{F} 1$ & $\mathrm{Kn}$ & S \\
\hline Prunus spinosa $\mathrm{L}$. & - & - & - & - & - & - & - & + & $\mathrm{F} 2$ & Ap1 & S \\
\hline Pseudotsuga menziesi (Mirb.) Franco & + cul & - & - & - & - & - & - & - & F1 & Kn & \\
\hline Pyracantha coccinea M. Roem. & - & - & + cul & + cul & - & - & - & - & $\mathrm{F} 2$ & ErW & \\
\hline Quercus robur $\mathrm{L}$. & - & + cul & + cul & - & - & - & + cul & $+\mathrm{cul}$ & $\mathrm{F} 1$ & Ap2/Sp2 & $\mathrm{S}, \mathrm{F}$ \\
\hline Rhamnus cathartica $\mathrm{L}$. & - & - & + & - & - & - & - & - & $\mathrm{F} 2$ & $\mathrm{Ap} 1 / \mathrm{Sp} 1$ & \\
\hline Rhododendron catawbiense Michx. & - & - & + cul & - & - & - & - & - & $\mathrm{F} 2$ & ErW & \\
\hline Ribes alpinum L. & - & $1 \mathrm{cul}$ & - & - & - & - & + cul & - & $\mathrm{F} 2$ & ErW & \\
\hline Ribes sanguineum Pursh & - & - & - & - & - & + cul & - & - & $\mathrm{F} 2$ & ErW & \\
\hline Robinia pseudoacacia L. & - & + & 1 & - & + & - & + & - & $\mathrm{F} 1$ & $\mathrm{Kn}$ & S \\
\hline Rosa blanda Aiton & - & $1 \mathrm{cul}$ & - & - & - & - & - & - & $\mathrm{F} 2$ & ErW & $\mathrm{S}, \mathrm{F}$ \\
\hline Rosa canina L. & - & 1 & - & - & - & - & - & + & $\mathrm{F} 2$ & Ap1 & $S$ \\
\hline Rosa dumalis Bechst. emend. Boulenger & - & - & - & + & - & - & - & - & $\mathrm{F} 2$ & Ap1 & $S$ \\
\hline Rosa multiflora Thunb. & - & - & 1 cul & - & - & - & - & - & $\mathrm{F} 2$ & Kn & $S, F$ \\
\hline Rosa 'Poznań' & - & - & 1 cul & - & - & - & - & - & $\mathrm{F} 2$ & ErW & S \\
\hline Rosa majalis $\times R$. rugosa & - & - & - & $1 \mathrm{cul}$ & - & - & - & - & $\mathrm{F} 2$ & ErW & $\mathrm{S}, \mathrm{F}$ \\
\hline Rosa $\times$ rugotida Belder \& Wijnands & - & - & - & $1 \mathrm{cul}$ & - & - & - & - & $\mathrm{F} 2$ & ErW & $\mathrm{S}, \mathrm{F}$ \\
\hline Sambucus nigra L. & - & + & 1 & + & - & + & - & + & $\mathrm{F} 2$ & Ap1 & S \\
\hline Sorbus aucuparia L. emend. Hedl. & - & - & - & - & - & - & + & - & $\mathrm{F} 1$ & $\mathrm{Ap} 1 / \mathrm{Sp} 1$ & $S$ \\
\hline Sorbus intermedia (Ehrh.) Pers. & - & - & - & + cul & - & - & - & - & $\mathrm{F} 1$ & ErG & S \\
\hline Spiraea 'Arguta' & - & + cul & - & + cul & - & - & - & - & $\mathrm{F} 2$ & ErW & \\
\hline Spiraea chamedrifolia L. emend. Jacq. & - & - & - & - & + cul & - & - & - & $\mathrm{F} 2$ & ErW & $\mathrm{F}$ \\
\hline Spiraea $\times$ vanhouttei (Briot) Zabel & - & $2 \mathrm{cul}$ & $1 \mathrm{cul}$ & + cul & + cul & - & - & - & $\mathrm{F} 2$ & ErW & \\
\hline Symphoricarpos albus (L.) S.F. Blanke & - & $2 \mathrm{cul}$ & 2 cul & $1 \mathrm{cul}$ & $1 \mathrm{cul}$ & - & - & - & $\mathrm{F} 2$ & ErW & $\mathrm{F}$ \\
\hline Symphoricarpos $\times$ chenaultii Rehder & - & - & $1 \mathrm{cul}$ & $1 \mathrm{cul}$ & - & - & - & - & $\mathrm{F} 2$ & ErW & \\
\hline Symphoricarpos orbicularis Moench & - & - & $1 \mathrm{cul}$ & - & - & - & - & - & $\mathrm{F} 2$ & ErW & \\
\hline Syringa vulgaris $\mathrm{L}$. & + cul & - & + cul & + cul & + cul & - & - & + cul & $\mathrm{F} 2$ & ErW & $\mathrm{S}, \mathrm{F}$ \\
\hline Taxus baccata $\mathrm{L}$. & - & - & + cul & $1 \mathrm{cul}$ & + cul & - & - & - & $\mathrm{F} 2$ & ErG & $\mathrm{S}, \mathrm{F}$ \\
\hline Thuja orientalis $\mathrm{L}$. & + cul & - & - & - & - & - & - & - & $\mathrm{F} 1$ & ErW & $\mathrm{S}, \mathrm{F}$ \\
\hline Tilia 'Euchlora' & - & - & - & - & - & + cul & - & - & $\mathrm{F} 1$ & ErW & $\mathrm{S}, \mathrm{F}$ \\
\hline Tilia cordata Mill. & - & + & + & - & - & + & - & - & $\mathrm{F} 1$ & Ap2 & $\mathrm{S}, \mathrm{F}$ \\
\hline Tilia platyphyllos Scop. & + cul & - & - & - & - & - & - & - & F1 & ErG & $\mathrm{S}, \mathrm{F}$ \\
\hline Ulmus laevis Pall. & - & + & - & - & - & - & - & - & F1 & Ap2 & $S$ \\
\hline Viburnum lantana L. & - & - & + cul & - & - & - & - & - & $\mathrm{F} 2$ & ErW & S \\
\hline Viburnum rhytidophyllum Hemsl. & - & - & - & - & + cul & - & - & - & $\mathrm{F} 2$ & ErW & S \\
\hline Weigela floryda (Bunge) A. DC. & - & - & - & - & - & + cul & - & - & $\mathrm{F} 2$ & ErW & \\
\hline \multicolumn{12}{|c|}{ Herb layer } \\
\hline Acer campestre L. & - & - & - & - & $\mathrm{R}$ & - & - & - & F1 & Ap2/Sp2 & $S$ \\
\hline Acer negundo L. & - & - & - & - & - & - & - & $\mathrm{R}$ & F1 & $\mathrm{Kn}$ & S \\
\hline Acer platanoides L. & - & + & - & - & - & - & - & 1 & F1 & Ap1 & S \\
\hline
\end{tabular}




\begin{tabular}{|c|c|c|c|c|c|c|c|c|c|c|c|}
\hline \multirow[b]{2}{*}{ Species } & \multicolumn{8}{|c|}{ Cemetery } & \multirow[b]{2}{*}{ LF } & \multirow[b]{2}{*}{$\mathrm{GH}$} & \multirow{2}{*}{$\begin{array}{c}\text { Symbolic } \\
(\mathrm{S}) \text { and } \\
\text { funeral }(\mathrm{F}) \\
\text { plants }\end{array}$} \\
\hline & I & II & III & IV & $\mathrm{V}$ & VI & VII & VIII & & & \\
\hline Acer pseudoplatanus L. & 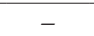 & - & - & - & - & - & - & + & F1 & Ap1 & $\mathrm{S}$ \\
\hline Achillea millefolium L. s.s. & $\mathrm{R}$ & + & 1 & + & + & - & 1 & - & $\mathrm{H}$ & Ap1 & \\
\hline Aegopodium podagraria $\mathrm{L}$. & + cul & $1 \mathrm{cul}$ & + cul & $+\mathrm{cul}$ & + cul & - & - & $3 \mathrm{cul}$ & $\mathrm{H}$ & Ap2/Sp2 & S, F \\
\hline Aethusa cynapium L. & $\mathrm{R}$ & - & - & - & - & - & - & - & $\mathrm{T} 1$ & Ar & \\
\hline Agrimonia eupatoria L. & - & - & - & - & - & - & - & $\mathrm{R}$ & $\mathrm{H}$ & Ap1 & \\
\hline Agrostis capillaris L. & - & 1 & - & - & - & - & - & - & $\mathrm{H}$ & Ap1 & \\
\hline Alcea rosea L. & R cul & - & - & - & - & - & - & - & $\mathrm{H}$ & ErG & S \\
\hline Alliaria petiolata (M. Bieb) Cavara \& Grande & - & + & - & - & - & - & - & 2 & $\mathrm{~T} 2$ & Ap1 & \\
\hline Allium oleraceum $\mathrm{L}$. & - & - & - & - & - & - & - & + & G & Ap1 & S \\
\hline Allium scorodoprasum L. & $2 \mathrm{cul}$ & - & $1 \mathrm{cul}$ & $1 \mathrm{cul}$ & $1 \mathrm{cul}$ & $1 \mathrm{cul}$ & - & $2 \mathrm{cul}$ & G & Ar & $S$ \\
\hline Allium vineale $\mathrm{L}$. & + & 1.1 & 1.1 & + & $\mathrm{R}$ & - & - & + & G & Ap1 & S \\
\hline Anchusa officinalis L. & $\mathrm{R}$ & - & - & - & - & - & - & - & $\mathrm{H}$ & Ap1 & \\
\hline Anemone nemorosa $\mathrm{L}$. & - & - & $+\mathrm{cul}$ & - & - & - & - & + cul & G & Sp2 & S \\
\hline Anemone ranunculoides $\mathrm{L}$. & - & - & + cul & $+\mathrm{cul}$ & $+\mathrm{cul}$ & - & - & + cul & G & Sp2 & $S, F$ \\
\hline Anthemis ruthenica M. Bieb. & - & - & $\mathrm{R}$ & - & - & - & - & - & $\mathrm{T} 1$ & $\mathrm{Ar}$ & \\
\hline Arabis caucasica Schltdl. in Willd. & + cul & - & - & - & - & - & - & - & $\mathrm{H}$ & ErW & \\
\hline Arenaria serpyllifolia L. & - & - & - & - & - & - & + & - & $\mathrm{T} 1$ & Ap1 & \\
\hline $\begin{array}{l}\text { Arrhenatherum elatius (L.) P. Beauv. ex J. Presl \& C. } \\
\text { Presl }\end{array}$ & - & - & - & - & - & - & - & + & $\mathrm{H}$ & Ap1 & \\
\hline Artemisia vulgaris $\mathrm{L}$. & $\mathrm{R}$ & + & $\mathrm{R}$ & - & - & $\mathrm{R}$ & + & + & $\mathrm{H}$ & Ap1 & S \\
\hline Aster novi-belgii L. & - & - & - & - & - & - & - & + cul & $\mathrm{H}$ & $\mathrm{Kn}$ & $\mathrm{F}$ \\
\hline Atriplex patula $\mathrm{L}$. & - & + & - & - & - & - & - & + & $\mathrm{T} 1$ & Ap1 & \\
\hline Ballota nigra $\mathrm{L}$. & + & + & $\mathrm{R}$ & - & - & $\mathrm{R}$ & - & + & $\mathrm{H}$ & $\mathrm{Ar}$ & \\
\hline Bellis perennis $\mathrm{L}$. & 1 & - & 1 & + & 1 & - & 1 & + & $\mathrm{H}$ & Ap1 & S \\
\hline Berteroa incana (L.) DC. & + & - & - & - & - & - & + & + & $\mathrm{T} 2$ & Ap1 & \\
\hline Bidens frondosa $\mathrm{L}$. & + & - & - & - & - & - & - & - & $\mathrm{T} 1$ & $\mathrm{Kn}$ & \\
\hline Brassica napus L. & - & - & $\mathrm{R}$ & - & - & - & - & - & $\mathrm{T} 1$ & ErG & \\
\hline Bromus carinatus Hook. \& Arn. & - & - & - & - & - & - & - & + & $\mathrm{H}$ & $\mathrm{Kn}$ & \\
\hline Bromus inermis Leyss. & - & - & - & - & - & - & + & - & $\mathrm{H}$ & Ap1 & \\
\hline Bromus sterilis L. & - & - & - & - & - & - & - & + & $\mathrm{T} 2$ & $\mathrm{Ar}$ & \\
\hline Calluna vulgaris (L.) Hull & - & - & + cul & - & - & - & - & - & $\mathrm{C}$ & Sp1 & \\
\hline Campanula rapunculoides $\mathrm{L}$. & + & - & + cul & - & - & - & - & + cul & $\mathrm{H}$ & Ap1 & $S, F$ \\
\hline Capsella bursa-pastoris (L.) Medik. & $\mathrm{R}$ & - & + & + & + & + & + & + & $\mathrm{T} 1$ & $\mathrm{Ar}$ & \\
\hline Cardamine hirsuta L. & - & - & $\mathrm{R}$ & - & - & $\mathrm{R}$ & - & - & $\mathrm{T} 1$ & Ap1 & \\
\hline Carex hirta $\mathrm{L}$. & - & + & - & - & - & - & - & - & G & Ap1 & \\
\hline Carex spicata Huds. & - & + & - & + & + & - & - & - & $\mathrm{H}$ & Ap1 & \\
\hline Cerastium glomeratum Thuill. & - & - & - & - & - & $\mathrm{R}$ & - & - & $\mathrm{T} 1$ & Ap1 & \\
\hline Cerastium holostoides Fr. emend. Hyl. & - & - & $\mathrm{R}$ & - & - & - & - & - & $\mathrm{H}$ & Ap1 & \\
\hline Cerastium semidecandrum L. & + & - & - & - & - & - & - & - & $\mathrm{H}$ & Ap1 & \\
\hline Chaerophyllum temulum $\mathrm{L}$. & + & - & - & - & - & - & - & + & $\mathrm{T} 2$ & Ap1/Sp1 & \\
\hline Chelidonium majus L. & + & + & $\mathrm{R}$ & $\mathrm{R}$ & $\mathrm{R}$ & - & - & + & $\mathrm{H}$ & Ap1 & S \\
\hline Chenopodium album $\mathrm{L}$. & - & + & $\mathrm{R}$ & - & - & $\mathrm{R}$ & - & - & $\mathrm{T} 1$ & Ap1 & \\
\hline Chenopodium hybridum L. & + & - & - & - & - & - & - & - & $\mathrm{T} 1$ & $\mathrm{Ar}$ & \\
\hline Chenopodium strictum Roth & - & - & $\mathrm{R}$ & - & - & - & - & - & $\mathrm{T} 1$ & $\mathrm{Kn}$ & \\
\hline Cichorium intybus L. & + & + & - & $\mathrm{R}$ & - & $\mathrm{R}$ & - & $\mathrm{R}$ & $\mathrm{H}$ & $\mathrm{Ar}$ & \\
\hline Cirsium arvense (L.) Scop. & - & - & - & - & - & - & - & $\mathrm{R}$ & G & Ap1 & S \\
\hline Cirsium vulgare (Savi) Ten. & - & - & - & - & - & $\mathrm{R}$ & - & - & $\mathrm{T} 2$ & Ap1 & S \\
\hline Convolvulus arvensis L. & - & - & + & $\mathrm{R}$ & - & - & + & + & G & Ap1 & S \\
\hline Conyza canadensis (L.) Cronquist & + & + & + & 1 & $\mathrm{R}$ & $\mathrm{R}$ & + & + & $\mathrm{T} 1$ & $\mathrm{Kn}$ & \\
\hline Coronilla varia $\mathrm{L}$. & - & - & - & - & - & - & - & + & $\mathrm{H}$ & Ap1 & \\
\hline Crepis biennis $\mathrm{L}$. & - & - & - & - & - & - & - & $\mathrm{R}$ & $\mathrm{T} 2$ & Ap1 & \\
\hline Crocus chrysanthus (Herb) Herb. & R cul & - & - & - & - & - & - & - & G & ErW & S \\
\hline Crocus vernus (L.) Hill & - & - & $+\mathrm{cul}$ & - & - & - & - & - & $\mathrm{G}$ & ErW & $S$ \\
\hline Dactylis glomerata L. & - & + & - & $\mathrm{R}$ & + & $\mathrm{R}$ & - & 1 & $\mathrm{H}$ & Ap1 & \\
\hline Dactylis polygama Horv. & $\mathrm{R}$ & + & - & - & - & - & - & 1 & $\mathrm{H}$ & Sp1 & \\
\hline Daucus carota $\mathrm{L}$. & - & - & - & - & - & $\mathrm{R}$ & - & - & $\mathrm{T} 2$ & Ap1 & \\
\hline Diplotaxis muralis (L.) DC. & - & - & $\mathrm{R}$ & - & - & - & - & - & $\mathrm{T} 1$ & Kn & \\
\hline Echinochloa crus-galli (L.) P. Beauv. & - & - & - & - & - & - & $\mathrm{R}$ & - & $\mathrm{T} 1$ & $\mathrm{Ar}$ & \\
\hline Elymus caninus (L.) L. & - & - & - & - & - & - & - & + & G & Ap1 & \\
\hline Elymus repens (L.) Gould & + & + & + & - & - & + & + & + & G & Ap1 & \\
\hline Equisetum arvense $\mathrm{L}$. & $\mathrm{R}$ & - & - & - & - & - & - & - & G & Ap1 & \\
\hline Erigeron annuus (L.) Pers. & - & $\mathrm{R}$ cul & - & - & - & - & - & - & $\mathrm{T} 2$ & $\mathrm{Kn}$ & $\mathrm{F}$ \\
\hline
\end{tabular}




\begin{tabular}{|c|c|c|c|c|c|c|c|c|c|c|c|}
\hline \multirow[b]{2}{*}{ Species } & \multicolumn{8}{|c|}{ Cemetery } & \multirow[b]{2}{*}{ LF } & \multirow[b]{2}{*}{$\mathrm{GH}$} & \multirow{2}{*}{$\begin{array}{c}\text { Symbolic } \\
\text { (S) and } \\
\text { funeral (F) } \\
\text { plants }\end{array}$} \\
\hline & I & II & III & IV & V & VI & VII & VIII & & & \\
\hline $\begin{array}{l}\text { Erigeron ramosus (Walters) Britton, Sterns \& } \\
\text { Poggenb. }\end{array}$ & R cul & - & - & - & - & - & - & - & $\mathrm{T} 2$ & $\mathrm{Kn}$ & $\mathrm{F}$ \\
\hline Erodium cicutaria (L.) L’Hér. & - & - & + & $\mathrm{R}$ & - & - & - & - & $\mathrm{T} 1$ & Ap1 & \\
\hline Erophila verna (L.) Chevell. & - & - & - & - & - & - & + & - & $\mathrm{T} 1$ & Ap1 & \\
\hline Euphorbia helioscopia L. & - & - & $\mathrm{R}$ & - & - & - & - & - & $\mathrm{T} 1$ & $\mathrm{Ar}$ & \\
\hline Euphorbia peplus L. & - & - & $\mathrm{R}$ & $\mathrm{R}$ & - & - & - & - & $\mathrm{T} 1$ & $\mathrm{Ar}$ & \\
\hline Falcaria vulgaris Bernh. & - & - & - & - & - & - & + cul & $1 \mathrm{cul}$ & $\mathrm{H}$ & Ap1 & \\
\hline Fallopia dumetorum (L.) Holub & $\mathrm{R}$ & - & - & - & - & - & - & - & $\mathrm{T} 1$ & Ap1/Sp1 & \\
\hline Festuca rubra L. s.s. & 1.1 & - & - & - & - & - & - & - & $\mathrm{H}$ & Ap1 & \\
\hline Ficaria verna Huds. & $1 \mathrm{cul}$ & $1 \mathrm{cul}$ & 2 cul & $3 \mathrm{cul}$ & 3 cul & $1 \mathrm{cul}$ & + cul & $3 \mathrm{cul}$ & G & Ap2/Sp2 & \\
\hline Gagea arvensis (Pers.) Dumort. & $1 \mathrm{cul}$ & + cul & - & $1 \mathrm{cul}$ & $2 \mathrm{cul}$ & - & + cul & + cul & G & Ar & $\mathrm{F}$ \\
\hline Gagea lutea (L.) Ker Gawl. & $1 \mathrm{cul}$ & - & $1 \mathrm{cul}$ & $3 \mathrm{cul}$ & $3 \mathrm{cul}$ & $1 \mathrm{cul}$ & + cul & 2 cul & G & Sp2 & $\mathrm{F}$ \\
\hline Gagea pratensis (Pers.) Dumort. & 2 & 3 & 3 & 3 & 3 & 1 & 2 & 1 & G & Ap2 & \\
\hline Galanthus nivalis L. & + cul & - & - & $1 \mathrm{cul}$ & R cul & - & - & + cul & G & Ap2 & S, F \\
\hline Galeopsis pubescens Besser & + & - & - & - & - & - & - & - & $\mathrm{T} 1$ & Ap1/Sp1 & \\
\hline Galinsoga ciliata (Raf.) S.F. Blake & + & - & $\mathrm{R}$ & $\mathrm{R}$ & - & - & - & - & $\mathrm{T} 1$ & $\mathrm{Kn}$ & \\
\hline Galinsoga parviflora Cav. & + & + & + & $\mathrm{R}$ & - & - & - & - & $\mathrm{T} 1$ & $\mathrm{Kn}$ & \\
\hline Galium mollugo L. s.s. & + & - & - & - & - & - & - & - & $\mathrm{H}$ & Ap1 & \\
\hline Geranium molle $\mathrm{L}$. & - & - & + & $\mathrm{R}$ & - & - & - & - & $\mathrm{T} 1$ & Kn & \\
\hline Geranium pusillum Burm. F. ex L. & $\mathrm{R}$ & - & $\mathrm{R}$ & $\mathrm{R}$ & $\mathrm{R}$ & $\mathrm{R}$ & $\mathrm{R}$ & - & $\mathrm{T} 1$ & $\mathrm{Ar}$ & \\
\hline Geranium pratense L. & - & - & - & - & - & - & - & + & $\mathrm{H}$ & Ap1 & \\
\hline Geum urbanum $\mathrm{L}$. & - & + & - & - & - & - & - & + & $\mathrm{H}$ & Ap1 & \\
\hline Glechoma hederacea L. & + & + & + & + & + & + & - & - & $\mathrm{H}$ & Ap1 & \\
\hline Hedera helix L. & - & + cul & - & - & - & - & - & $1 \mathrm{cul}$ & $\mathrm{C}$ & Kn & $\mathrm{S}, \mathrm{F}$ \\
\hline Helichrysum arenarium (L.) Moench & - & - & - & - & - & - & $\mathrm{R}$ & - & $\mathrm{H}$ & Ap1 & \\
\hline Hemerocallis citrina Baroni & - & - & $1 \mathrm{cul}$ & - & - & - & - & - & $\mathrm{H}$ & ErW & \\
\hline Hemerocallis fulva L. & - & - & + cul & - & - & - & - & + & $\mathrm{H}$ & ErW & F \\
\hline Hieracium pilosella L. & + & - & - & - & - & - & - & - & $\mathrm{H}$ & Ap1 & \\
\hline Holosteum umbellatum $\mathrm{L}$. & - & - & - & - & - & - & + & - & $\mathrm{T} 1$ & Ap1 & \\
\hline Hordeum murinum L. & - & - & $\mathrm{R}$ & $\mathrm{R}$ & - & - & - & $\mathrm{R}$ & $\mathrm{T} 2$ & Ar & \\
\hline Hyoscyanus niger L. & - & - & $\mathrm{R}$ & - & - & - & - & - & $\mathrm{T} 1$ & $\mathrm{Ar}$ & \\
\hline Impatiens parviflora $\mathrm{DC}$. & $\mathrm{R}$ & - & - & - & - & - & - & + & $\mathrm{T} 1$ & $\mathrm{Kn}$ & \\
\hline Juglans regia $\mathrm{L}$. & $\mathrm{R}$ & - & - & - & - & - & - & - & $\mathrm{F} 1$ & $\mathrm{Kn}$ & \\
\hline Lactuca serriola $\mathrm{L}$. & - & $\mathrm{R}$ & - & - & - & - & - & $\mathrm{R}$ & $\mathrm{T} 2$ & $\mathrm{Ar}$ & \\
\hline Lamium album $\mathrm{L}$. & + cul & + cul & + cul & $\mathrm{R}$ cul & R cul & $1 \mathrm{cul}$ & - & $1 \mathrm{cul}$ & $\mathrm{H}$ & $\mathrm{Ar}$ & $\mathrm{F}$ \\
\hline Lamium purpureum $\mathrm{L}$. & + & - & + & - & - & $\mathrm{R}$ & + & + & $\mathrm{T} 1$ & $\mathrm{Ar}$ & \\
\hline Lapsana communis L. s.s. & - & - & + & - & - & - & - & + & $\mathrm{T} 1$ & Ap1/Sp1 & \\
\hline Lavendula angustifolia Mill. & - & - & + cul & - & - & - & - & - & $\mathrm{C}$ & ErW & S \\
\hline Leontodon autumnalis $\mathrm{L}$. & $\mathrm{R}$ & - & $\mathrm{R}$ & $\mathrm{R}$ & - & - & - & - & $\mathrm{H}$ & Ap1 & \\
\hline Lobelia erinus L. & - & - & + cul & - & - & - & - & - & T0 & Er0 & \\
\hline Lolium multiflorum Lam. & - & - & 1 & - & - & 4 & - & - & $\mathrm{H}$ & $\mathrm{Kn}$ & \\
\hline Lolium perenne $\mathrm{L}$. & 1 & 2 & 2 & 2 & 3 & 1 & 2 & + & $\mathrm{H}$ & Ap1 & \\
\hline Malva neglecta Wallr. & $\mathrm{R}$ & - & $\mathrm{R}$ & + & - & $\mathrm{R}$ & + & - & $\mathrm{T} 2$ & $\mathrm{Ar}$ & \\
\hline Malva pusilla Sm. & - & - & - & - & - & $\mathrm{R}$ & - & - & $\mathrm{T} 2$ & $\mathrm{Ar}$ & \\
\hline Malva sylvestris $\mathrm{L}$. & - & - & - & - & - & $\mathrm{R}$ & - & - & $\mathrm{T} 2$ & $\mathrm{Ar}$ & \\
\hline Medicago falcata L. & - & - & - & - & - & - & + & - & $\mathrm{H}$ & Ap1 & \\
\hline Melandrium album (Mill.) Garcke & + & - & - & - & - & - & - & $\mathrm{R}$ & $\mathrm{H}$ & Ap1 & \\
\hline Mentha longifolia (L.) L. & + cul & - & - & - & - & - & - & - & $\mathrm{H}$ & Ap2 & \\
\hline Moehringia trinervia (L.) Clairv. & + & - & - & - & - & - & - & + & $\mathrm{H}$ & Ap1/Sp1 & \\
\hline Muscari botryoides (L.) Mill. & + cul & $\mathrm{R}$ cul & + cul & - & - & - & - & - & G & ErW & F \\
\hline Myosostis sylvatica Ehrh. ex Hoffm. & $+\mathrm{cul}$ & - & - & - & - & - & - & - & $\mathrm{T} 1$ & ErG & $S, F$ \\
\hline Narcissus poëticus L. & - & $\mathrm{R}$ cul & - & - & - & - & - & - & G & ErW & S, F \\
\hline Narcissus pseudonarcissus L. & - & $\mathrm{R}$ cul & - & - & - & - & - & - & G & ErW & S, F \\
\hline Onopordon acanthium L. & - & - & $\mathrm{R}$ & - & - & - & - & - & $\mathrm{T} 2$ & $\mathrm{Ar}$ & \\
\hline Ornithogalum boucheanum Asch. & $2 \mathrm{cul}$ & - & - & - & - & - & - & - & G & ErG & $\mathrm{F}$ \\
\hline Ornithogalum nutans L. & - & - & - & - & - & $1 \mathrm{cul}$ & - & - & G & ErG & $\mathrm{F}$ \\
\hline Ornithogalum umbellatum $\mathrm{L}$. & $1 \mathrm{cul}$ & $1 \mathrm{cul}$ & $2 \mathrm{cul}$ & + cul & $1 \mathrm{cul}$ & + cul & + cul & $1 \mathrm{cul}$ & G & ErG & $\mathrm{F}$ \\
\hline Oxalis dillenii Jacq. & + & - & + & + & + & - & - & - & $\mathrm{T} 1$ & $\mathrm{Kn}$ & \\
\hline Oxalis corniculata L. $\times$ O. dilenii Jacq. & + & - & - & - & - & - & - & - & $\mathrm{T} 1$ & $\mathrm{Kn} \times \mathrm{Kn}$ & \\
\hline Pachysandra terminalis Siebold \& Zucc. & - & - & + cul & - & - & - & - & - & $\mathrm{C}$ & ErW & \\
\hline Papaver rhoeas L. & - & - & $\mathrm{R}$ & - & - & - & - & - & $\mathrm{T} 1$ & $\mathrm{Ar}$ & S \\
\hline Papaver somniferum $\mathrm{L}$. & $\mathrm{R}$ & - & - & - & - & - & - & - & $\mathrm{T} 1$ & Ef & S \\
\hline Phalaris arundinacea $\mathrm{L}$. & - & - & - & - & - & - & - & $\mathrm{R}$ & $\mathrm{H}$ & Ap1 & \\
\hline
\end{tabular}




\begin{tabular}{|c|c|c|c|c|c|c|c|c|c|c|c|}
\hline \multirow[b]{2}{*}{ Species } & \multicolumn{8}{|c|}{ Cemetery } & \multirow[b]{2}{*}{ LF } & \multirow[b]{2}{*}{$\mathrm{GH}$} & \multirow{2}{*}{$\begin{array}{c}\text { Symbolic } \\
\text { (S) and } \\
\text { funeral (F) } \\
\text { plants }\end{array}$} \\
\hline & I & II & III & IV & $\mathrm{V}$ & VI & VII & VIII & & & \\
\hline Picris hieraciodes L. & $\mathrm{R}$ & - & - & - & - & - & - & - & $\mathrm{H}$ & Ap1 & \\
\hline Plantago lanceolata $\mathrm{L}$. & + & + & + & + & - & 1 & 1 & - & $\mathrm{H}$ & Ap1 & \\
\hline Plantago major L. s.s. & + & 1 & + & 1 & - & - & - & - & $\mathrm{H}$ & Ap1 & \\
\hline Poa annua $\mathrm{L}$. & + & - & - & 1 & 1 & 1 & + & + & $\mathrm{T} 2$ & Ap1 & \\
\hline Poa nemoralis $\mathrm{L}$. & - & 1 & - & - & - & - & - & 1 & $\mathrm{H}$ & Apl/Sp1 & \\
\hline Poa pratensis L. s.s. & + & - & 2 & - & 1 & 2 & 2 & + & $\mathrm{H}$ & Ap1 & \\
\hline Polygonum aviculare $\mathrm{L}$. & 1 & + & 1 & 2 & - & - & + & - & $\mathrm{T} 1$ & Ap1 & \\
\hline Polygonum persicaria $\mathrm{L}$. & $\mathrm{R}$ & - & - & - & - & - & - & - & $\mathrm{T} 1$ & Ap1 & \\
\hline Populus alba L. & - & - & + & - & - & - & - & - & F1 & Ap1 & \\
\hline Potentilla argentea L. s.s. & - & - & + & - & - & - & + & + & $\mathrm{H}$ & Ap1 & \\
\hline Potenilla reptans $\mathrm{L}$. & $\mathrm{R}$ & - & - & + & - & - & - & - & $\mathrm{H}$ & Ap1 & \\
\hline Puschkinia scilloides Adams & - & - & $1 \mathrm{cul}$ & - & - & - & - & - & G & ErG & $\mathrm{F}$ \\
\hline Ranunculus repens L. & - & + & - & - & - & - & - & - & $\mathrm{H}$ & Ap1 & \\
\hline Robinia pseudoacia L. & + & - & + & - & - & - & - & - & $\mathrm{F} 1$ & Kn & $S$ \\
\hline Rubus caesius L. & - & - & - & - & - & - & - & + & $\mathrm{C}$ & Ap1/Sp1 & \\
\hline Rumex crispus L. & - & + & - & - & - & - & - & $\mathrm{R}$ & $\mathrm{H}$ & Apl & \\
\hline Rumex obtusifolius L. & - & + & - & - & - & $\mathrm{R}$ & $\mathrm{R}$ & - & $\mathrm{H}$ & Ap1 & \\
\hline Rumex thyrsiflorus Fingerh. & + & - & - & - & - & - & - & - & $\mathrm{H}$ & Ap1 & \\
\hline Sambucus nigra L. & $\mathrm{R}$ & $\mathrm{R}$ & - & - & - & - & - & - & $\mathrm{F} 2$ & Ap1 & \\
\hline Saponaria officinalis L. & - & - & - & - & - & - & + cul & $1 \mathrm{cul}$ & G & Ap1 & $\mathrm{F}$ \\
\hline Scilla sibirica Haw. & - & - & $1 \mathrm{cul}$ & R cul & R cul & - & - & $+\mathrm{cul}$ & G & ErG & $S, F$ \\
\hline Scleranthus perennis L. & - & - & - & - & - & - & $\mathrm{R}$ & - & $\mathrm{H}$ & Ap1 & \\
\hline Sedum acre L. & - & - & - & - & - & - & + & - & $\mathrm{H}$ & Ap1 & \\
\hline Sedum reflexum L. & - & - & $+\mathrm{cul}$ & - & - & - & - & - & $\mathrm{H}$ & $\mathrm{Sp} 2$ & \\
\hline Setaria viridis (L.) P. Beauv. & - & - & + & - & - & - & + & - & $\mathrm{T} 1$ & $\mathrm{Ar}$ & \\
\hline Silene vulgaris (Moench) Garcke & - & - & - & - & - & - & + & - & $\mathrm{H}$ & Ap1 & \\
\hline Solanum nigrum L. emend. Mill. & + & - & $\mathrm{R}$ & - & - & - & - & - & $\mathrm{T} 1$ & $\mathrm{Ar}$ & \\
\hline Solidago canadensis L. & - & - & - & - & + cul & - & - & R cul & $\mathrm{G}$ & $\mathrm{Kn}$ & \\
\hline Solidago gigantea Aiton & - & $\mathrm{R}$ cul & - & - & - & - & - & R cul & G & $\mathrm{Kn}$ & \\
\hline Sonchus oleraceus L. & + & - & $\mathrm{R}$ & $\mathrm{R}$ & - & $\mathrm{R}$ & - & - & $\mathrm{T} 1$ & $\mathrm{Ar}$ & \\
\hline Stellaria media (L.) Vill. & + & - & 1 & + & + & + & + & + & $\mathrm{T} 1$ & Ap1 & \\
\hline Stellaria pallida (Dumort.) Piré & 1 & - & 1 & + & + & + & + & 1 & $\mathrm{~T} 2$ & Ap1 & \\
\hline Tanacetum vulgare $\mathrm{L}$. & - & - & - & - & - & - & - & + & $\mathrm{H}$ & Ap1 & \\
\hline Taraxacum officinale Web. & 1 & 1 & 1 & 1 & 1 & 1 & 2 & + & $\mathrm{H}$ & Ap1 & \\
\hline Tilia cordata Mill. & + & - & - & - & - & + & - & - & $\mathrm{F} 1$ & Ap1 & \\
\hline Torilis japonica (Houtt.) DC. & - & - & - & - & - & - & - & + & $\mathrm{T} 2$ & Ap1 & \\
\hline Trifolium campestre Schreb. & $\mathrm{R}$ & - & - & - & - & - & - & - & $\mathrm{T} 1$ & Ap1 & \\
\hline Trifolium pretense L. & $\mathrm{R}$ & + & - & - & - & - & - & - & $\mathrm{H}$ & Ap1 & \\
\hline Trifolium repens L. & + & + & 1 & + & + & + & 2 & - & $\mathrm{H}$ & Ap1 & \\
\hline Tulipa gesneriana L. & - & - & $+\mathrm{cul}$ & - & - & - & - & $+\mathrm{cul}$ & G & ErW & $\mathrm{F}$ \\
\hline Tulipa sylvestris L. & $1 \mathrm{cul}$ & - & $2 \mathrm{cul}$ & - & - & $1 \mathrm{cul}$ & - & - & G & ErG & $\mathrm{F}$ \\
\hline Ulmus laevis Pall. & - & - & $\mathrm{R}$ & $\mathrm{R}$ & - & - & - & - & $\mathrm{F} 1$ & Ap1/Sp1 & \\
\hline Urtica dioica L. & $\mathrm{R}$ & - & - & - & - & - & + & 1 & $\mathrm{H}$ & Ap1/Sp1 & \\
\hline Urtica urens L. & - & - & - & $\mathrm{R}$ & - & - & - & - & $\mathrm{T} 1$ & $\mathrm{Ar}$ & \\
\hline Verbascum lychnitis L. & $\mathrm{R}$ & - & - & - & - & - & - & - & $\mathrm{T} 2$ & Ap1 & \\
\hline Veronica arvensis L. & $\mathrm{R}$ & - & - & - & - & - & + & - & $\mathrm{T} 1$ & $\mathrm{Ar}$ & \\
\hline Veronica chamaedrys L. s.s & - & + & + & $\mathrm{R}$ & $\mathrm{R}$ & - & - & + & $\mathrm{H}$ & Ap1 & \\
\hline Veronica persica Poir. & - & - & - & - & - & $\mathrm{R}$ & - & - & $\mathrm{T} 1$ & $\mathrm{Kn}$ & \\
\hline Veronica polita Fr. & - & - & - & - & - & $\mathrm{R}$ & - & - & $\mathrm{T} 1$ & $\mathrm{Ar}$ & \\
\hline Veronica sublobata M.A. Fisch. & 1 & 1 & 2 & 1 & 1 & 1 & 1 & 1 & $\mathrm{~T} 1$ & Ap1 & \\
\hline Veronica triphyllos L. & - & - & - & - & - & - & + & - & $\mathrm{T} 1$ & $\mathrm{Ar}$ & \\
\hline Vinca major L. & - & - & $1 \mathrm{cul}$ & - & - & - & - & - & $\mathrm{H}$ & ErW & \\
\hline Vinca minor L. & - & - & - & - & - & - & - & $1 \mathrm{cul}$ & $\mathrm{C}$ & ErW & $S, F$ \\
\hline Viola cyanea Č́lak. & - & - & + cul & - & - & - & - & $1 \mathrm{cul}$ & $\mathrm{H}$ & $\mathrm{Kn}$ & $S, F$ \\
\hline Viola odorata L. & $1 \mathrm{cul}$ & $1 \mathrm{cul}$ & $1 \mathrm{cul}$ & $+\mathrm{cul}$ & $+\mathrm{cul}$ & $\mathrm{R}$ cul & + cul & $1 \mathrm{cul}$ & $\mathrm{H}$ & $\mathrm{Kn}$ & $S, F$ \\
\hline
\end{tabular}

FC (frequency classes): R - 1-2 specimens, + - several specimens, $1-$ very rare (covering $1-5 \%$ of cemetery area excluding graves), 2 - rare (5-25\%), 3 - moderately frequent (25-50\%), 4 - frequent (50-75\%), 5 - very frequent (75-100\%); cul - cultivated, introduced in the past or presently introduced to cultivation.

LF (life-forms): F1 - megaphanerophytes, F2 - nanophanerophytes, C - chamaephytes, G - geophytes, H - hemicryptophytes, T1 - annual therophytes, T2 - biennial therophytes, T0 - non-wintering therophytes; li - climber, pp - parasite, ppep - semi-parasite.

GH (geographical-historical status): spontaneously occurring spontaneophytes (Sp1), planted spontaneophytes (Sp2), semi-synanthropic spontaneophytes $(\mathrm{Ap} / \mathrm{Sp})$, autapophytes (Ap1) - species that penetrated spontaneously from natural localities to localities changed by human activity, hemeroapophytes (Ap2) - cultivated native species, archaeophytes (Ar), kenophytes (Kn), ephemerophytes (Ef), vegetatively spreading ergasiophytes (ErW), generatively spreading ergasiophytes (ErG), non-wintering ergasiophytes (Er0). 
Table 3. Numbers of species included in frequency classes in park-like old cemeteries in Poznań

\begin{tabular}{lrrrrrrrrr}
\hline Frequency class & \multicolumn{1}{c}{ I } & II & \multicolumn{1}{c}{ III } & \multicolumn{1}{c}{ IV } & V & VI & VII & VIII & Total \\
\hline $\mathrm{R}$ & 26 & 7 & 23 & 18 & 9 & 20 & 6 & 12 & 121 \\
+ & 59 & 41 & 56 & 36 & 27 & 16 & 45 & 61 & 341 \\
1 & 28 & 25 & 64 & 32 & 20 & 18 & 14 & 26 & 227 \\
2 & 6 & 10 & 11 & 5 & 4 & 2 & 7 & 6 & 51 \\
3 & - & - & 1 & 5 & 6 & - & 1 & 3 & 16 \\
4 & - & - & - & - & - & 1 & - & - & 1 \\
5 & - & - & - & - & - & 1 & - & - & 1 \\
Total & 119 & 83 & 155 & 96 & 66 & 58 & 73 & 108 & 758 \\
\hline
\end{tabular}

them, in general the most diverse were megaphanerophytes (101 species) and hemicryptophytes (67). Also in individual cemeteries megaphanerophytes were the most numerous, except for cemeteries VII and VIII, where hemicryptophytes dominated. In contrast, many annual therophytes were recorded in cemeteries I (26) and III (26) (Table 4).

Interestingly, also the proportion of geophytes was relatively high (31 species). Those plants can survive in unfavourable conditions thanks to deeply hidden buds, protected against drought and low temperature, which increases their chance to survive in: cemeteries. This group includes primarily bulb plants flowering in spring, e.g. Gagea arvensis, G. lutea, Galanthus nivalis, Muscari botryoides, Narcissus poëticus, N. pseudonarcissus, Ornithogalum boucheanum, O. nutans, O. umbellatum, Puschkinia scilloides, Scilla sibirica, Tulipa gesnerana, and T. sylvestris.

Out of the 315 taxa recorded in this study, native species (apophytes and spontaneophytes) accounted for more than $50 \%$. However, in individual cemeteries their proportion varied from $33 \%$ (cemetery VI) to $67 \%$ (cemetery III). In all cemeteries, apophytes were represented by Acer platanoides, Bellis perennis, Ficaria verna, Fraxinus excelsior, Gagea pratenis, Lolium perenne, Tanacetum vulgare, Taraxacum officinale, Ulmus laevis, and Veronica sublobata. Among alien plants, the largest number of species belonged to the group of ergasiophytes (71 species), followed by kenophytes (56) (Table 5). In all cemeteries, kenophytes were represented by Conyza canadensis, Ornithogalum umbellatum and Viola odorata. The smallest numbers of

Table 4. Numbers of species included in plant life-form categories in park-like old cemeteries in Poznań

\begin{tabular}{lrrrrrrrrr}
\hline Life-form & \multicolumn{1}{c}{ I } & \multicolumn{1}{c}{ II } & \multicolumn{1}{c}{ III } & \multicolumn{1}{c}{ IV } & V & VI & VII & VIII & Total \\
\hline F1 & 31 & 25 & 53 & 34 & 19 & 14 & 14 & 27 & 217 \\
F2 & 6 & 12 & 26 & 15 & 11 & 4 & 3 & 6 & 83 \\
C & 3 & 2 & 4 & 1 & 2 & 1 & 1 & 4 & 18 \\
G & 13 & 11 & 15 & 11 & 13 & 8 & 8 & 19 & 98 \\
H & 34 & 23 & 26 & 17 & 13 & 13 & 29 & 34 & 189 \\
T1 & 26 & 6 & 25 & 14 & 6 & 11 & 14 & 8 & 110 \\
T2 & 7 & 3 & 5 & 4 & 2 & 7 & 4 & 10 & 42 \\
T0 & - & - & 1 & - & - & - & - & - & 1 \\
Total & 120 & 82 & 155 & 96 & 66 & 58 & 73 & 108 & 758 \\
\hline
\end{tabular}

Table 5. Numbers of species included in geographical-historical groups in park-like old cemeteries in Poznań

\begin{tabular}{lrrrrrrrrr}
\hline \multicolumn{1}{c}{ Group } & \multicolumn{1}{c}{ I } & II & III & IV & V & VI & VII & VIII & Total \\
\hline Sp1 & 1 & 1 & 1 & 1 & 0 & 0 & 0 & 1 & 5 \\
Sp2 & 4 & 4 & 5 & 3 & 2 & 1 & 1 & 3 & 23 \\
Sp1/Ap1 & 5 & 3 & 4 & 3 & 1 & 2 & 2 & 7 & 27 \\
Sp2/Ap2 & 5 & 8 & 14 & 7 & 6 & 3 & 3 & 3 & 49 \\
Ap1 & 39 & 23 & 31 & 27 & 17 & 26 & 33 & 46 & 232 \\
Ap2 & 8 & 8 & 9 & 5 & 6 & 6 & 6 & 7 & 55 \\
Ar & 14 & 5 & 17 & 11 & 6 & 9 & 10 & 9 & 81 \\
Kn & 20 & 12 & 25 & 16 & 9 & 6 & 5 & 20 & 113 \\
Kn $\times$ Kn & 1 & 0 & 0 & 0 & 0 & 0 & 0 & 0 & 1 \\
Ef & 1 & 0 & 0 & 0 & 0 & 0 & 0 & 0 & 1 \\
ErW & 13 & 17 & 36 & 18 & 13 & 4 & 11 & 8 & 120 \\
ErG & 8 & 2 & 11 & 5 & 6 & 1 & 2 & 5 & 39 \\
ErG $\times$ Ap1 & 0 & 0 & 1 & 0 & 0 & 0 & 0 & 0 & 1 \\
ErO & 0 & 0 & 1 & 0 & 0 & 0 & 0 & 0 & 1 \\
Total & 119 & 83 & 155 & 96 & 66 & 58 & 73 & 108 & 758 \\
\hline
\end{tabular}

species of ergasiophytes were recorded in cemeteries VII (5) and VI (6), because their herbaceous layer was completely transformed by destruction of the former ground cover and sowing of new lawns.

Funeral plants are represented in the studied cemeteries by 74 species. The best indicator plants of cemeteries (including geophytes, distinguished by underlining) are Aster novi-belgii, Aesculus carnea, A. hippocastanum, Campanula rapunculoides, Caragana arborescens, Erigeron annuus, E. ramosus, Fagus sylvatica 'Atropurpurea', F. sylvatica 'Pendula', Fraxinus excelsior 'Pendula', Gagea arvensis, G. lutea, Galanthus nivalis, Hedera helix, Hemerocallis fulva, Lamium album, Ligustrum vulgare, Lonicera tatarica, Mahonia aquifolium, Muscari botryoides, Myosotis sylvatica, Narcissus poëticus, N. pseudonarcissus, Ornithogalum boucheanum, O. nutans, O. umbellatum, Parthenocissus quinquefolia, Philadelphus coronarius, Platanus $\times$ hispanica, Ribes alpinum, Salix alba 'Tristis', Saponaria officinalis, Scilla sibirica, Sedum reflexum, Spiraea chamaedrifolia, Symphoricarpos albus, Syringa vulgaris, Thuja plicata, Tulipa gesneriana, T. sylvestris, Vinca minor, Viola cyanea, and $V$. odorata.

Description of the process of synanthropization by means of indices allows to determine its rate and various aspects of anthropogenic transformations of flora. The following indices were analysed in this study according to CHMIEL (2006).

a) indices of flora synanthropization: WSc $=168 \%$, $\mathrm{WSt}=212 \%(\mathrm{WSc}=$ total synanthropization index, WSt $=$ permanent synanthropization index)

b) indices of apophytization: WAPc $=75.6 \%$, WAPt $=87.7 \%$ (WAPc $=$ total apophytization index, WAPt $=$ permanent apophytization index)

c) indices of flora anthropophytization: WANc $=92.5 \%$, WANt $=87.7 \%$ (WANc $=$ total anthropophytization index, WANt $=$ permanent anthropophytization index)

d) indices of flora archaeophytization: WARc = $19.5 \%$, WARt $=31.8 \%($ WARc $=$ total archaeo- 
phytization index, WARt $=$ permanent archaeophytization index)

e) indices of flora kenophytization: $\mathrm{WKNc}=36.7 \%$, $\mathrm{WKNt}=55.5 \%(\mathrm{WKNc}=$ total kenophytization index, WKNt = permanent kenophytization index)

f) flora modernization index: $\mathrm{WM}=6.3 \%$

g) index of floristic fluctuations: WF $=0.25 \%$.

On the basis of the diagram of cemetery similarity, three groups of cemeteries were distinguished (Fig. 1). One group is composed of cemeteries I (near Świętojańska St.), VII (near Kosynierów Square), and VIII (near Winogrady St.), the similar second group consists of cemeteries IV (near Królowej Jadwigi St.), V (near Grunwaldzka St.), and VI (near Ogrodowa St.), and the third, least similar group comprises cemeteries II (near Ostrobramska St.) and III (near Towarowa St.).

\section{DISCUSSION}

The dendroflora of cemeteries and parks has been studied by many researchers. In comparison to other habitats explored floristically, cemeteries and parks that were formerly cemeteries have a rich dendroflora, characteristic of cemeteries.

Parks that were formerly cemeteries, because of the species richness and age of the dendroflora, are valuable because of their nature and history. The most common species of woody plants, found in all the investigated cemeteries, are Acer platanoides and Fraxinus excelsior. Floristic species richness is the highest in cemetery III (155 species), and the lowest in cemetery VI (58 species).

Konon et al. (2005), who studied the dendroflora of six cemeteries in Lednica Landscape Park, recorded 23 species of trees and shrubs: 19 broad-leaved and four coniferous. In comparison, the dendroflora of eight parks that were formerly cemeteries includes 61 species of trees and 91 species in the shrub layer. In research on four active cemeteries in Poznań (Czarna et al. 2011), 89 species of trees and shrubs were found. It is noteworthy that in the urban cemeteries the number of species of trees and shrubs is much higher than in the village cemeteries. Similar disproportions were reported by KARCZMARZ \& TRZASKOWSKA (2013).

The flora of old cemeteries in Poznań is clearly distinguished from the flora of medieval fortified settlements (CelKa 1999), flora of Poznań (JACKOWIAK 1990), and flora of the eastern part of Gniezno Lakeland (CHмiel 2006) by its high indices of synanthropization, apophytization, anthropophytization, kenophytization, and archaeophytization, whereas indices of floristic fluctuations and flora modernization are very low.
A taxon new to the Polish flora is Rosa 'Poznan'. Roses as a genus were frequently used for planting in old cemeteries (CZARnA 1999).

It is noteworthy that a native species recorded in six cemeteries - Aegopodium podagraria - had a symbolic meaning: it symbolized faith because of the shape of its leaves It was certainly planted in cemeteries as an ornamental groundcover plant (KossaK 2017).

Results of this study confirm the hypothesis that many funeral plants are geophytes (17 species). The most interesting among them is Tulipa sylvestris. Besides, funeral plants include 26 species of trees $(7.8 \%$ of the total), 31 species of shrubs $(9.3 \%)$, and 33 herbaceous species $(9.9 \%)$.

\section{ACKNOWLEDGEMENTS}

I am grateful to Prof. Jerzy Zieliński (Institute of Dendrology in Kórnik) for verification and identification of the collected herbarium specimens of trees and shrubs. The study was supported by the $\mathrm{Na}-$ tional Science Centre in Kraków, Poland (grant no. NN304204937).

\section{REFERENCES}

Borcz Z. (2002): Elementy projektowania zieleni. Wydawnictwo Akademii Rolniczej we Wrocławiu, Wrocław.

CelKa Z. (1999): Rośliny naczyniowe grodzisk Wielkopolski. Prace Zakładu Taksonomii Roślin Uniwersytetu im. Adama Mickiewicza w Poznaniu 9. Bogucki Wydawnictwo Naukowe, Poznań.

Chмiel J. (2006): Zróżnicowanie przestrzenne flory jako podstawa ochrony przyrody $\mathrm{w}$ krajobrazie rolniczym. Prace Zakładu Taksonomii Roślin Uniwersytetu im. Adama Mickiewicza w Poznaniu 14. Bogucki Wydawnictwo Naukowe, Poznań.

CZARna A. (1999): Flora naczyniowa cmentarzy ewangelickich w Koźminie i Koźmińcu (Nizina Wielkopolska). Roczniki AR w Poznaniu 334, Botanika 4: 27-37.

Czarna A. (2005): Flora naczyniowa starego cmentarza katolickiego na Świerczewie w Poznaniu. Rocznik Naukowy Polskiego Towarzystwa Ochrony Przyrody „Salamandra” 9: 61-76.

CZARnA A. (2009): Rośliny naczyniowe środkowej Wielkopolski. Wydawnictwo Uniwersytetu Przyrodniczego w Poznaniu, Poznań.

CzARna A. (2016): Vascular plant flora on Cytadela Cemeteries in Poznań. Acta Agrobotanica 69(4): 1-17. http://dx.doi.org/10.5586/aa1695.

Czarna A., Woźnicka A., Maj M., Morozowska M. (2011): Flora of vascular plants of selected Poznań cemeteries. Acta Agrobotanica 64(4): 123-140. http://dx.doi.org/10.5586/aa.2011.054. 
DĄBSKi M., Oleś A. (2006): Analiza dendrologiczna zabytkowego cmentarza przy ulicy Lipowej w Lublinie. In: J. Rylke (ed.). Przyroda i miasto. T. 8. Wydawnictwo SGGW, Warszawa.

GAWRYŚ W. (2008): Słownik roślin zielnych łacińsko-polski. Officina Botanica, Kraków.

Hempelmann J. (1927): Die Praxis der Friedhofsgärterei. Anlage, Verwaltung und Instadhaltung von Friedhöfen und Gräbern. Parey, Berlin.

JACKOWIAK B. (1990): Antropogeniczne przemiany flory roślin naczyniowych Poznania. Uniwersytet im. Adama Mickiewicza w Poznaniu, seria Biologia 42: 1-232.

Karczmarz K., TrZaskowsKa E. 2013. Analiza dendroflory założeń cmentarnych $\mathrm{w}$ krajobrazie miasta i wsi Lubelszczyzny. Teka Komisji Architektury, Urbanistyki i Studiów Krajobrazowych PAN 9(4): 7-20.

Kobielus S. (2006): Florarium christianum. Symbolika roślin - chrześcijańska starożytność i średniowiecze. Tyniec Wydawnictwo Benedyktynów, Kraków.

Konon A., Krzyżaniak M., Urbański P. (2005): Stan cmentarzy poewangelickich na terenie Lednickiego Parku Krajobrazowego. Roczniki Akademii Rolniczej w Poznaniu 370, Ogrodnictwo 39: $45-51$.

Kopaliński W. (1985): Słownik mitów i tradycji kultury. Państwowy Instytut Wydawniczy, Warszawa.

Kossak S. (2017): O ziołach i zwierzętach. Wydawnictwo Marginesy, Warszawa.

Mirek Z., PięKoś-Mirkowa H., Zając A., Zając M. (2002): Flowering plants and pteridophytes of Poland: checklist. Biodiversity of Poland. Vol. 1. W. Szafer Institute of Botany, Polish Academy of Sciences, Kraków.

Niemirski W. (1973): Założenia programowe i normatywy techniczne projektowania. In: W. Niemirski:
Kształtowanie terenów zieleni. Wydawnictwo Arkady, Warszawa.

RutKowski L. (1998): Klucz do oznaczania roślin naczyniowych Polski niżowej. Wydawnictwo Naukowe PWN, Warszawa.

RYDZEWSKA A., KRZyŻANiAK M., URbaŃSKi P. (2011): Niegdyś sacrum, dziś profanum - dawne cmentarze ewangelickie Poznania i okolic. Niematerialne Wartości Krajobrazów Kulturowych. Prace Komisji Krajobrazu Kulturowego 15: 64-72.

Siewniak M., Mitkowska A. (1998): Tezaurus sztuki ogrodowej. Oficyna Wydawnicza Rytm, Warszawa.

SzuLCzewski J.W. (1951): Wykaz roślin naczyniowych w Wielkopolsce dotąd stwierdzonych. Poznańskie Towarzystwo Przyjaciół Nauk, Wydział Matematyczno-Przyrodniczy, Prace Komisji Biologicznej 12(6): 1-128.

TANAś S. (2008): Przestrzeń turystyczna cmentarzy. Wstęp do tanatoturystyki. Wydawnictwo Uniwersytetu Łódzkiego, Łódź.

Thellung A. (1915): Pflanzenwanderungen unter dem Einfluss des Menschen. Englers Botanische Jahrbucher, Lepizig 53(3-5), Beibl. nr 116: 37-68.

ZarzycKi K., TRZCiŃSKa-TaciK H., RóŻAŃSKa W., SZELĄG Z., Wotek J., Korzeniak U. (2002): Ecological indicator of vascular plants of Poland. W. Szafer Institute of Botany, Polish Academy of Sciences, Kraków.

ZióŁKowsKa M. (1988): Gawędy o drzewach. Ludowa Spółdzielnia Wydawnicza, Warszawa.

For citation: CZARNA A. (2017): Vascular flora of old cemeteries transformed into parks in Poznań. Steciana 21(3): 115-125. doi: 10.12657/ steciana.021.014 